\title{
Asymmetry: To make a distinction
}

\author{
Ari M.P. Koskinen \\ Department of Chemistry, University of Oulu, Linnanmaa, FIN-90570 OULU, FINLAND
}

\begin{abstract}
Amino acids provide a versatile starting point for the synthesis of enantiopure materials. In particular, chiral amino alcohols are useful building blocks for the synthesis of chiral azacrown ethers and cryptands. In this lecture, I shall review some of our recent findings on the syntheses of these compounds. The examples are drawn from on-going projects aiming at the syntheses of natural and non-natural compounds.
\end{abstract}

\section{INTRODUCTION}

Lasalocid $A$ and nonactin, two naturally occurring ionophore antibiotics (1), are examples of 'crown ether like' compounds which selectively bind alkali metal ions. This binding property is widely used in the design of new materials for macrocyclic hosts, and also provides convenient point of entry into studying the energetics and structural

features intermolecular interactions between hosts

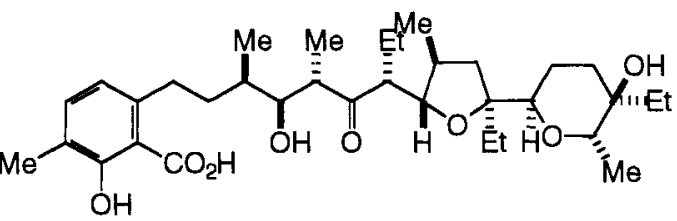

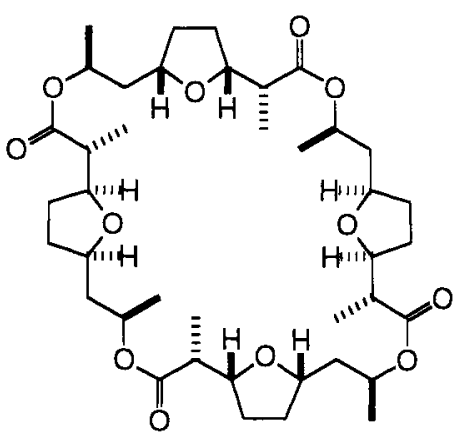
Lasalocid A

\section{Nonactin} and guests (2).

The cyclic nature of crown ethers is generally beneficial for the selective binding of these compounds with their guests. As exemplified by lasalocid $A$ and a number of other natural ionophores, this requirement is not an absolute one, if the conformation of the compound is restricted by some other structural features. In a series of papers, Still has examined the utility of chiral podands in the extraction of organic ammonium ions. The podands are conformationally rigid due to their hindered rotation around the $\mathrm{C}-\mathrm{C}$ bonds connecting the rings. Enantioselectivities in the extraction were found to be as high as $80 \%$ ee (3).

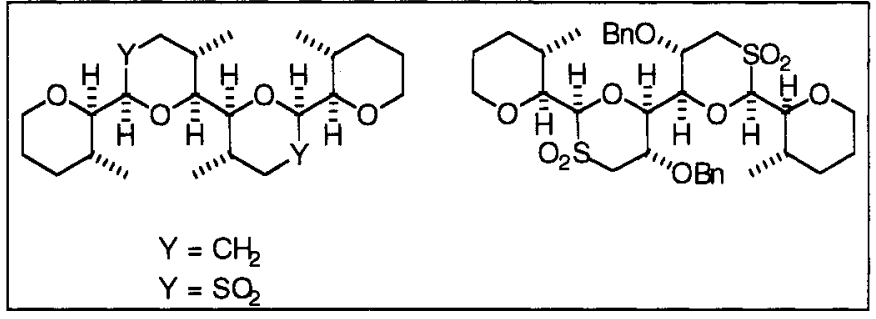


Chiral crown ethers as well as the corresponding $\mathrm{C}_{2}$-symmetrical cryptands, designed after Lehn's chiral host molecules (4) can in principle provide an environment which could function as a host for studying the energetics of intermolecular interactions between host and guest. The sites for functionality could be equipped with a number of probe substructures to shed light on e.g. dipolar, ionic and $\pi-\pi$ interactions.

chiral

The synthesis molecules

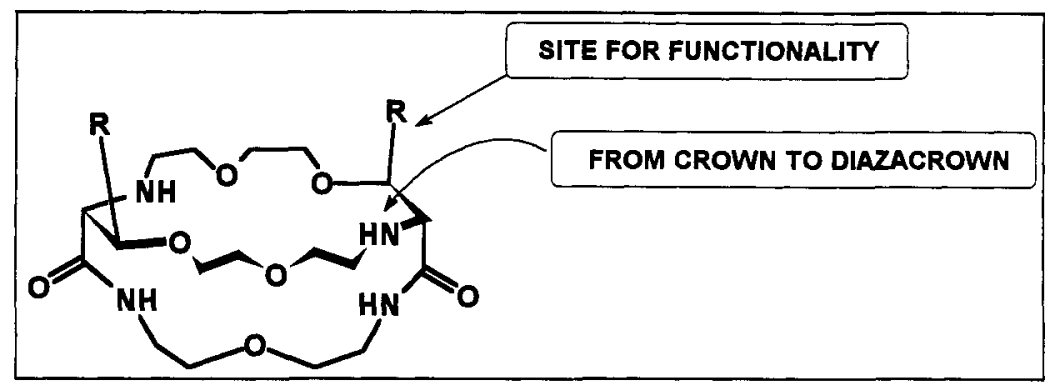

enantiopure form is of utmost importance in trying to elucidate the basic mechanisms of these three dimensional events. The past two decades have seen an enormous growth in the development of the technologies for asymmetric synthesis, and today, one can quite confidently say that most of the important stereochemical arrays can be synthesized in configurationally pure or at least highly enriched form (5). However, as with any synthetic technology, much remains to be done. The ideal of attaining the construction of multiple carbon carbon bonds with defined stereochemical relationships in even directly connected atoms is still far from universally achieved. The problem is much more complicated in cases where the stereogenic centers are far apart from each other, and not connected via a cyclic array.

\section{SYNTHESIS OF CHIRAL AMINO ALCOHOLS}

In this lecture, I would like to highlight some features of the synthesis of $\beta$-amino alcohols which are useful for the construction of rigid, stereochemically defined chiral cryptands such as the structure shown in the scheme below (6). These cryptands would provide a useful probe for studying the energetics involved in binding e.g. chiral ammonium ion guests, with auxiliary binding groups $(R)$ within the parent hosts.

The intermediate hydroxy amino acids and alcohols are also useful synthetic intermediates per se, as indicated below.

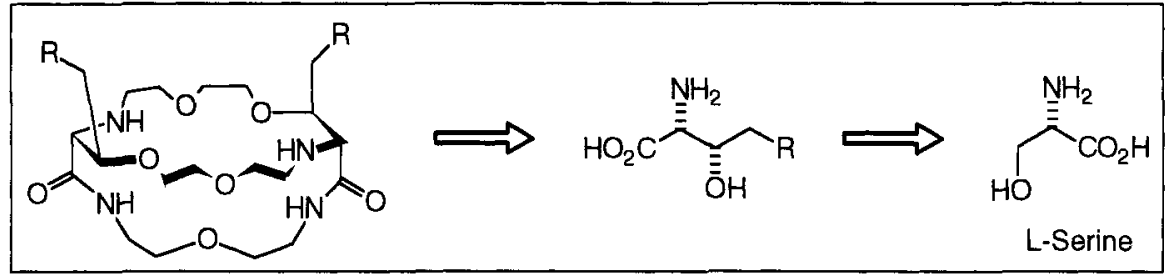

One of the most powerful general methods for the preparation of such serine-derived amino alcohols relies on a reagent controlled asymmetric allylation of the intermediate serinal derivative. Duthaler et al have shown that this reaction proceeds with practically complete reagent control (7).

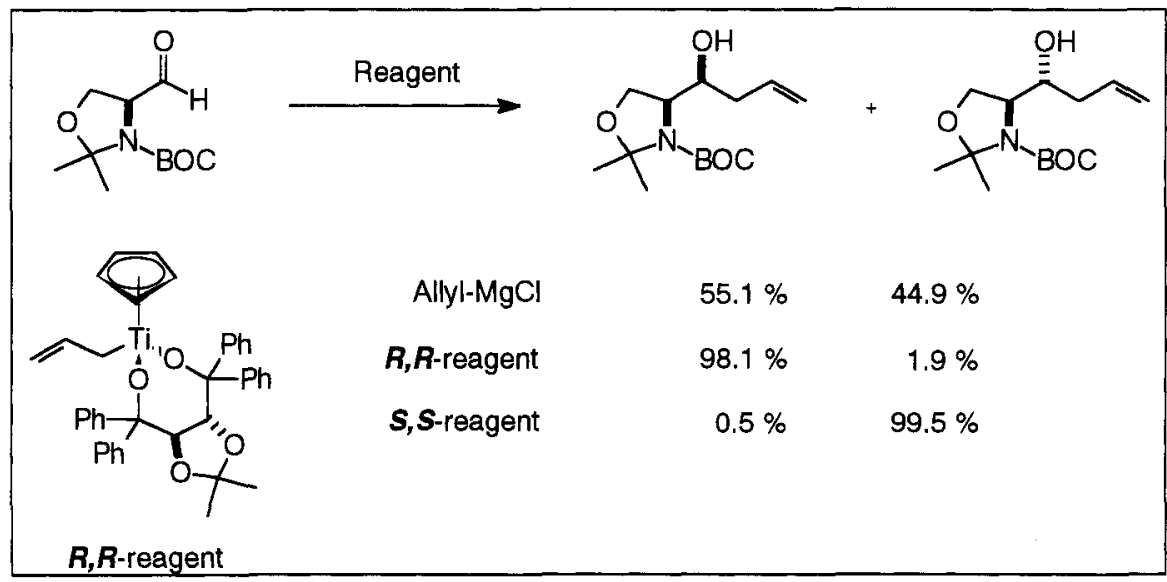


However, for our purposes, we wished to extend the $\gamma$-chiral $\beta$-ketophosphonate chemistry we have developed to allow access to a variety of consecutively functionalised carbon frameworks (8). Thus, our starting point was the conversion of serine into the phosphonate 2 in one step from the Garner ester 1 (9). Under carefully controlled conditions, this phosphonate can be converted, via a Horner-Wadsworth-Emmons reaction, into the enone $\underline{3}$ without loss of enantiopurity (10).

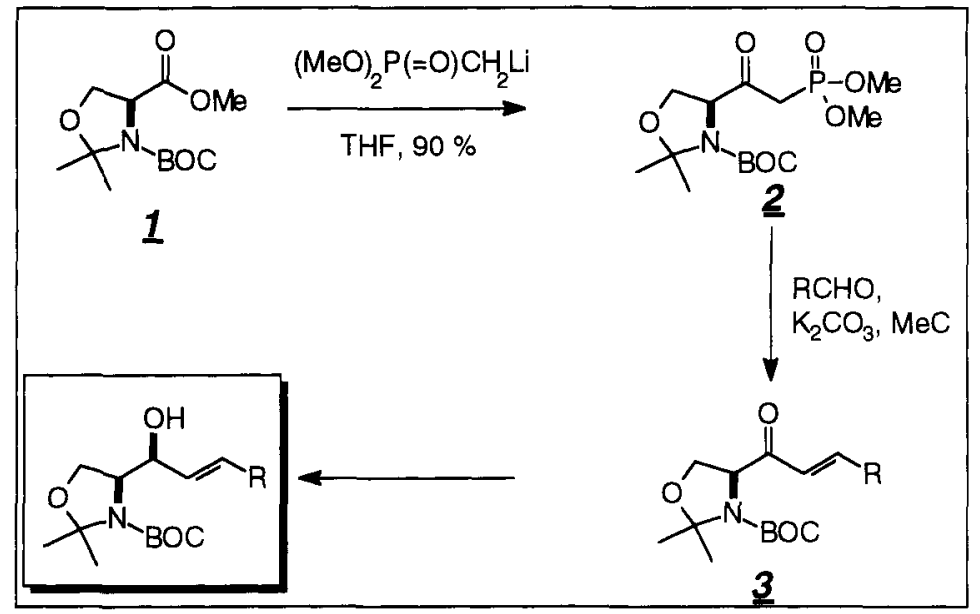

Diastereoselective reduction of such enones provides a rapid and efficient access into the aminodiols 4 , as evidenced by our synthesis of sphingosine bases (11).<smiles>CC1(C)OCC(C(=O)/C=C/I)N1C(=O)O[Na]</smiles>

$\underline{\mathbf{3}}$<smiles>[R]C=CC(O)C1COC(C)(C)N1C(=O)OC</smiles>

4a<smiles></smiles>

$\underline{4 b}$

synlanti Diastereomer ratios $(\underline{4 a}: \underline{4 b})$ in hydride reduction of $\mathbf{3}$

\begin{tabular}{|c|c|c|c|c|}
\hline Beductant & Solvent & $\mathrm{Ph}$ & $\begin{array}{l}R \\
\text { Et }\end{array}$ & $\underline{i-P r}$ \\
\hline L-Selectride & THF & $\overline{4: 1}$ & $2: 1$ & $\overline{3: 7}$ \\
\hline L-Selectride & $\mathrm{CH} 2 \mathrm{Cl} 2$ & $2: 1$ & $1.3: 1$ & $2: 1$ \\
\hline $\mathrm{NaBH} 4$ & $i-\mathrm{PrOH}$ & $1: 1$ & $3: 2$ & $3: 2$ \\
\hline $\mathrm{NaBH} 4$ & $\mathrm{MeOH}$ & $3: 1$ & $1: 1.2$ & $3: 2$ \\
\hline $\mathrm{NaBH} 4 / \mathrm{CeCl} 3$ & $i-\mathrm{PrOH}$ & $1: 2$ & $7: 4$ & $2: 1$ \\
\hline DIBAL & PhMe & $1: 6$ & & \\
\hline
\end{tabular}

For the final realisation of the synthesis of useful aminodiol synthons, we also required a practical method for the selective cleavage of the protecting groups, which eventually could be controlled by careful choice of reaction conditions. This has enabled us to extend this chemistry into the development of the syntheses of a number of amino-deoxysugars (12).

Following another line of strategy for the introduction of the aminoalcohol functionalities, we have utilised the Sharpless

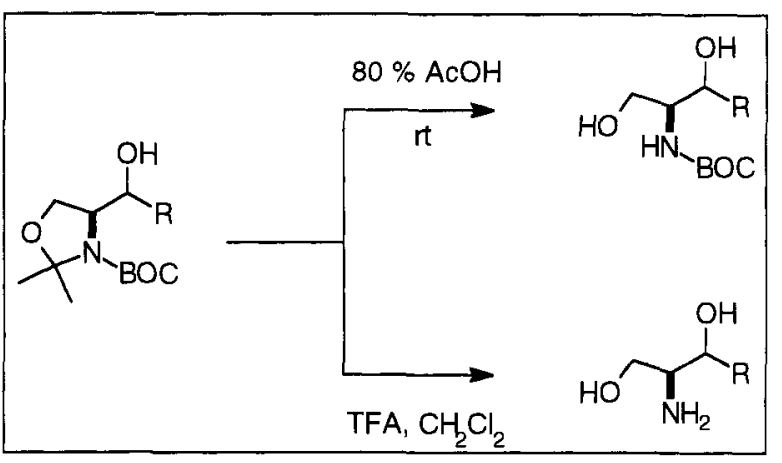
Asymmetric Dihydroxylation (AD) technology (13). Dihydroxylation of methyl cis-cinnamate was expected to proceed with low enantioselectivity. However, we were surprised to observe complete lack of enantioselectivity in this reaction under the standard AD conditions (assayed by chiral phase HPLC, less than $0.5 \%$ ee) (14). 
This finding prompted us to adopt a strategy based on the utilisation of methyl trans-cinnamate as the stating material. Conversion into the syn-amino alcohol would require inversion at the $\mathrm{C}_{2}$-center, which could be realised by opening the cyclic sulfate with bromide ion.

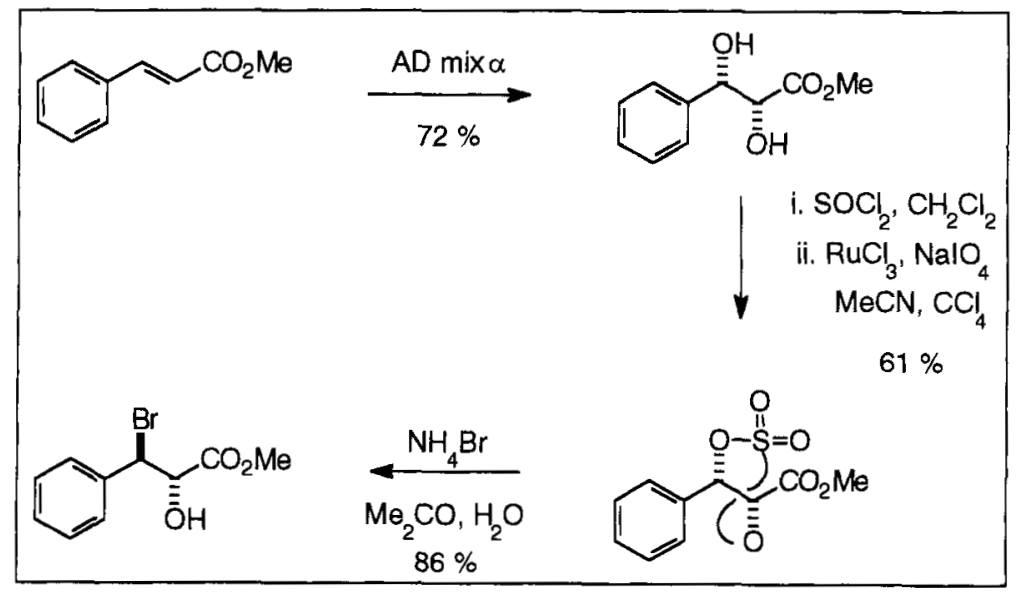

The bromo alcohol could then be converted to the corresponding azido alcohol simply by treatment with sodium azide. However, this led to a $4: 1$ mixture of diastereomers, presumably through the involvement of the corresponding epoxide. This problem could be readily alleviated by protecting the hydroxy group as the corresponding tert-butyl dimethyl silyl (TBS) ether.

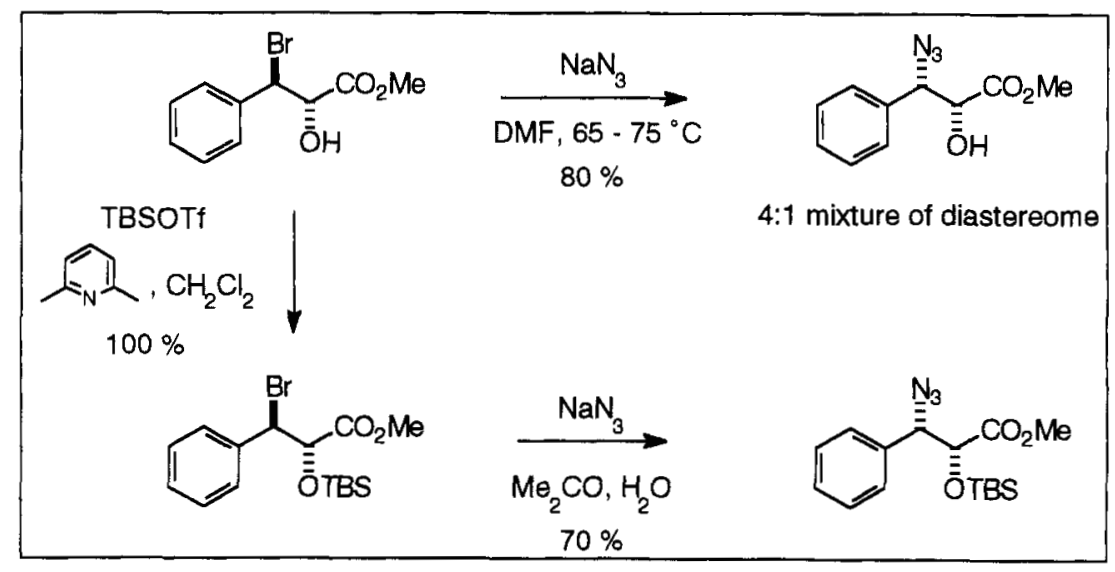
methods.

The azido alcohol was transformed into both the taxol and taxotere side chains using standard

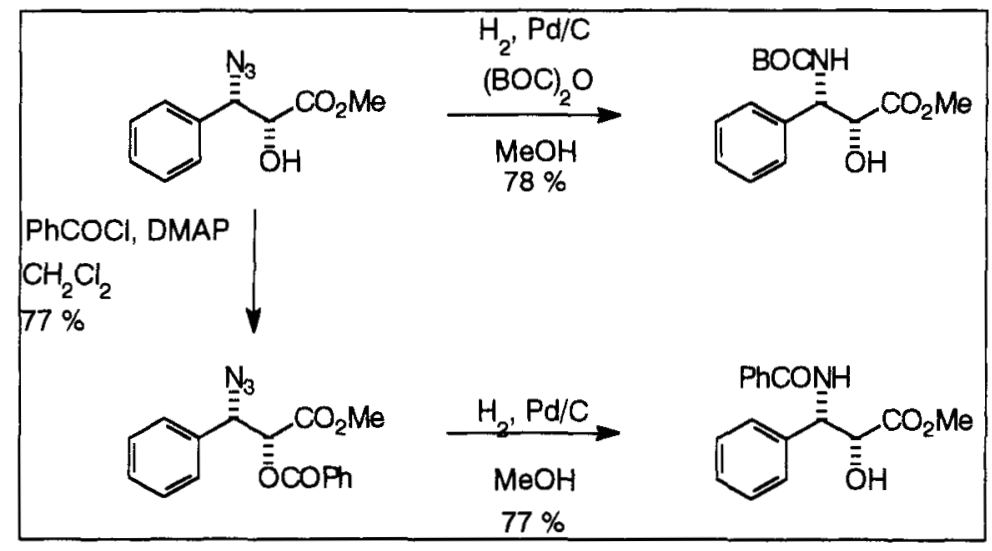


The versatility of the approach is also evident in the simple synthesis of the epi-taxotere side chain from the corresponding anti-azido alcohol.

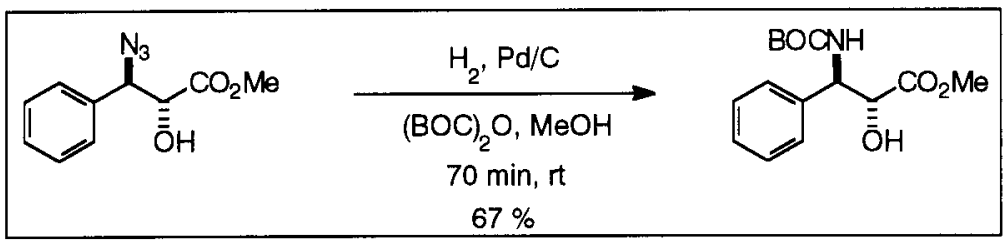

Another variation of the usefulness of the amino acid approach is shown by the short syntheses of phenylserine, diphenylserine and diphenylalanine (15). Grignard reaction of the Garner-Park aldehyde gave practically 1:1 mixture of the two diastereomers (only one shown in the following scheme), which could be separated by repeated fractional crystallisations. Cleavage of the protecting groups followed by selective oxidation of the primary alcohol then yielded the target phenylserine in enantiopure form.

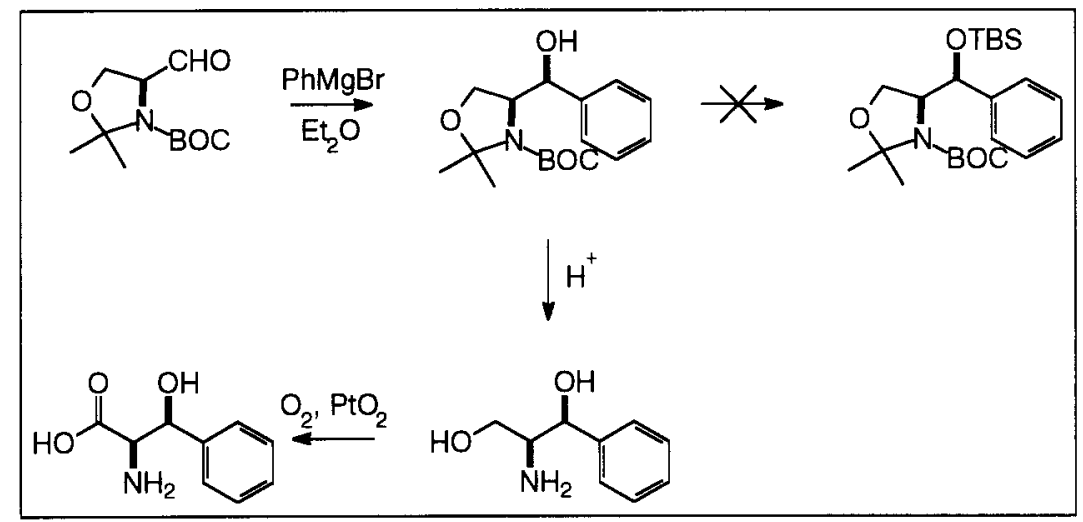

Finally, an extension of the utilisation of the Garner ester 1 has enabled us to develop a rapid entry into diphenylalanine, as well as the corresponding diphenylserine. Double Grignard reaction of the ester followed by deoxygenation and deprotection gave diphenylalanine. Alternatively, omission of the reductive step lead to diphenylserine. The former has been utilised in the synthesis of chiral ligands for catalytic asymmetric cyclopropanation (16).

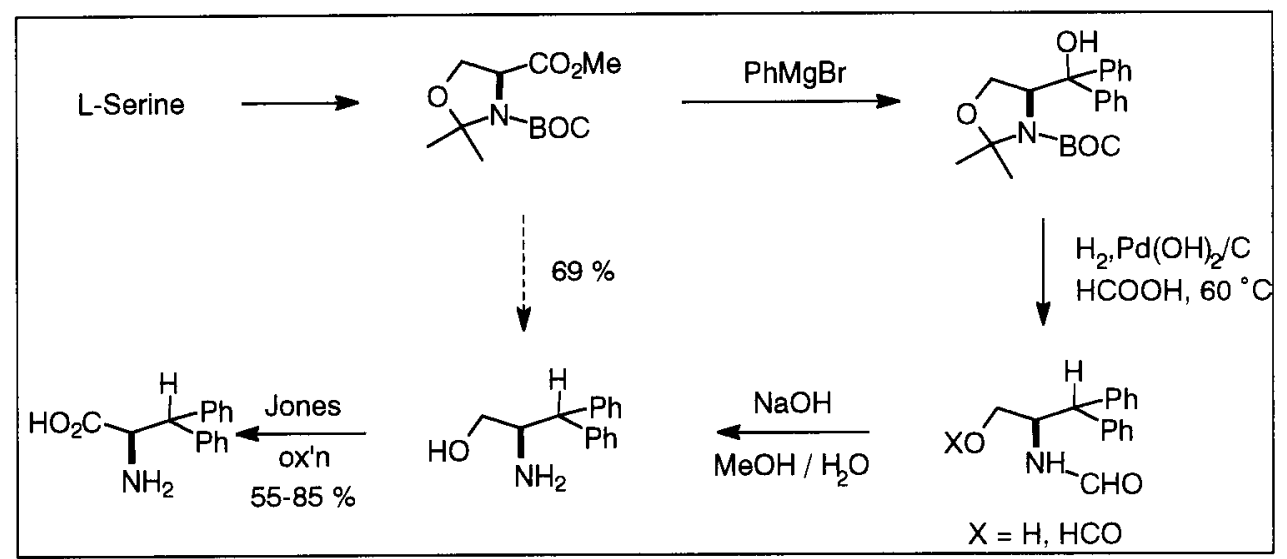


Acknowledgments: I would like to thank my very able co-workers for their invaluable contributions. They are: Dr Jingshan Chen, Dr Esko Karvinen, Mr Heikki Hassila, Mr Seppo Hiltunen, Mr Timo Kantola, Mrs Päivi Koskinen, Mr Vesa Myllymäki, Mrs Leena Otsomaa, and Mr Jussi Siirilä. I would also like to thank Professor Kari Rissanen, University of Joensuu, for the X-ray structural determinations. Helpful discussions with, and a generous gift of a chiral ligand by, Professor K. Barry M. Sharpless, The Scripps Research Institute, USA, is also gratefully acknowledged. Financially, our work has been generously supported by the Finnish Academy of Sciences, the Technology Development Centre (TEKES, Finland), Leiras Oy (Finland), Orion Corporation (Finland), the Nuffield Foundation (UK), Glaxo Group Research (UK) and the University of Oulu.

\section{References:}

1. (a) Polyether Antibiotics, Westley, J.W. (Ed.), Marcel Dekker, New York, 1982, vols. 1-2. (b) Masamune, S.; Bates, G.S.; Corcoran, J.W. Angew. Chem. 1977, 89, 602.

2. (a) Vögtle, F. Supramolecular Chemistry, John Wiley \& Sons, Chichester, 1991. (b) Dietrich, B.; Viout, P.; Lehn, J.-M. Macrocyclic Chemistry, Verlag Chemie, Weinheim, 1993. (c) Crown Compounds, Cooper, S.R. (Ed.), Verlag Chemie, Weinheim, 1992.

3. (a) Li, G.; Still, W.C. J. Org. Chem. 1991, 56, 6964; (b) Wang, X.; Erickson, S.D.; limori, T.; Still, W.C. J. Am. Chem. Soc. 1992, 114, 4128; (c) Tang, S.; Still, W.C. Tetrahedron Lett. 1993, 34, 6701.

4. Lehn, J.-M.; Potvin, P.G. Can. J. Chem. 1988, 66, 195.

5. (a) Koskinen, A.M.P. Asymmetric Synthesis of Natural Products, John Wiley \& Sons, Chichester, 1993. (b) Noyori, R. Asymmetric Catalysis in Organic Synthesis, John Wiley \& Sons, Chichester, 1993.

6. Koskinen, A.M.P. Pure Appl. Chem. 1992, 65, 1465.

7. Hafner, A.; Duthaler, R.O.; Marti, R.; Rihs, G.; Rothe-Streit, P.; Schwarzenbach, F. J. Am. Chem. Soc. 1992, 114, 2321.

8. Koskinen, A.M.P.; Krische, M.J. Synlett 1990, 665.

9. Garner, P.; Park, J.M. J. Org. Chem. 1987, 52, 2361.

10. Koskinen, A.M.P.; Koskinen, P.M. Synlett, 1993, 501.

11. Koskinen, A.M.P.; Koskinen, P.M. Tetrahedron Lett. 1993, 34, 6765.

12. Koskinen, A.M.P.; Otsomaa, L.A. manuscript in preparation.

13. (a) Sharpless, K.B.; Amberg, W.; Bennani, Y.; Crispino, G.; Hartung, J.; Jeong, K.; Kwong, H.; Morikawa, K.; Wang, Z.M.; Xu, D.; Zhang, X.L. J. Org. Chem. 1992, 57, 2768; (b) Wang, L.; Sharpless, K.B. J. Am. Chem. Soc. 1992, 114, 7568; (c) Morikawa, K.; Park, J.; Andersson, P.G.; Hashiyama, T.; Sharpless, K.B. J. Am. Chem. Soc. 1993, 115, 8463; for recent reviews, cf: (d) Sharpless, K.B. Tetrahedron 1994, 50, 4235. (e) Lohray, B.B. Tetrahedron: Asymmetry 1992, 3, 1317.

14. Koskinen, A.M.P.; Karvinen, E.K.; Sirilä. J.P. J. Chem. Soc., Chem. Commun. 1994, 21.

15. Koskinen, A.M.P.; Myllymäki, V. manuscript in preparation.

16. Koskinen, A.M.P.; Hassila, H. manuscript in preparation. 